\title{
E-RNAi: a web application to design optimized RNAi constructs
}

\author{
Zeynep Arziman, Thomas Horn and Michael Boutros*
}

\author{
Boveri-Group Signaling and Functional Genomics, German Cancer Research Center, \\ Im Neuenheimer Feld 580, D-69120 Heidelberg, Germany \\ Received February 14, 2005; Revised and Accepted April 13, 2005
}

\begin{abstract}
RNA interference (RNAi) has become a powerful genetic approach to systematically dissect gene function on a genome-wide scale. Owing to the penetrance and efficiency of RNAi in invertebrates, model organisms such as Drosophila melanogaster and Caenorhabditis elegans have contributed significantly to the identification of novel components of diverse biological pathways, ranging from early development to fat storage and aging. For the correct assessment of phenotypes, a key issue remains the stringent quality control of long double-stranded RNAs (dsRNA) to calculate potential off-target effects that may obscure the phenotypic data. We here describe a web-based tool to evaluate and design optimized dsRNA constructs. Moreover, the application also gives access to published predesigned dsRNAs. The E-RNAi web application is available at http://e-rnai.dkfz.de/.
\end{abstract}

\section{INTRODUCTION}

Until recently, systematic reverse genetic approaches to probe loss-of-function phenotypes have been difficult to conduct. This has been largely due to the limitations in the ability to generate genome-wide collections of directed knock-out mutants. However, the discovery of post-transcriptional silencing mechanisms by small interfering RNAs (siRNAs) has allowed the development of tools to efficiently knock down expression of specific genes. First discovered in Caenorhabditis elegans, double-stranded RNA (dsRNA) molecules have been shown in subsequent studies to represent both important endogenous regulators of gene expression and a powerful new tool to silence gene expression $(1,2)$. In C.elegans, several genome-scale collections of dsRNAs have been applied to study developmental defects, fat storage and other phenotypes (3-5), which has been facilitated by the ease of dsRNA introduction: feeding of worms with E.coli which express dsRNA molecules is sufficient to produce loss-of-function phenotypes. Although feeding dsRNA to Drosophila is not effective, the Drosophila system is tractable to RNAi in both embryos and cultured cells (6). Typically 300-700 bp of dsRNA are synthesized from DNA templates containing terminal $\mathrm{T} 7$ promoters and the resulting molecules are simply added to the culture medium. These dsRNA molecules are taken up by cells through an unknown transport mechanism and are intracellularly processed into functional 21 mer siRNAs. Several well-characterized cell lines of embryonic origin are available and have been used to perform genome-scale RNAi screens for various phenotypes (7-13).

The efficiency of RNAi by long dsRNAs in invertebrates is probably due to the 'natural' pooling of many $21 \mathrm{nt}$ sequences. Long dsRNAs are intracellularly cleaved into 21-22 nt siRNAs by the Dicer complex and direct the degradation of target mRNAs through the RNA-induced silencing complex (RISC) (2). Although a dsRNA should be designed to match to one specific gene, off-target effects can occur if siRNAs have sequence homology to genes that are not supposed to be targeted. Furthermore, the knock-down of target transcripts might differ depending on the efficiency of siRNAs derived from long dsRNAs. It has been proposed that parameters for siRNA efficiency include GC content, asymmetry and thermodynamic stability (14). In efficient siRNAs, the $5^{\prime}$ end of the anti-sense strand and the target site have relatively low thermodynamic stability whereas the $5^{\prime}$ end of the sense strand has a high thermodynamic stability. These thermodynamic properties appear to be important for promoting the incorporation of the anti-sense strand into the RISC, blocking the incorporation of the sense strand and promoting RISC-antisense strand mediated mRNA degradation (15-17). In order to design efficient and specific siRNAs for experiments in mammalian cells, a number of computational tools have been developed that incorporate recent design rules (18-20).

We have developed the E-RNAi web application to design and evaluate dsRNA constructs suitable for RNAi experiments in Drosophila and C.elegans. It can also be used for the design of enzymatically digested long dsRNA (esiRNAs) for mammalian cells (21). dsRNA sequences (RNAi probes)

*To whom correspondence should be addressed. Tel: +49 6221 421951; Fax: +49 6221 421959; Email: m.boutros@dkfz.de

(c) The Author 2005. Published by Oxford University Press. All rights reserved.

The online version of this article has been published under an open access model. Users are entitled to use, reproduce, disseminate, or display the open access version of this article for non-commercial purposes provided that: the original authorship is properly and fully attributed; the Journal and Oxford University Press are attributed as the original place of publication with the correct citation details given; if an article is subsequently reproduced or disseminated not in its entirety but only in part or as a derivative work this must be clearly indicated. For commercial re-use, please contact journals.permissions@oupjournals.org 
are evaluated for their predicted specificity and efficiency. Since DNA templates used to generate dsRNAs are generated by PCR, primer pairs suitable to amplify DNA templates from genomic DNA or cDNA are calculated. In addition E-RNAi allows access to predesigned dsRNAs from published experiments.

\section{WEB APPLICATION}

Our aim was to create a web application to automate the design of optimized dsRNA constructs that are commonly used for RNAi experiments in invertebrate model organisms such as Drosophila and C.elegans. To this end, the E-RNAi application has to accomplish several tasks, including (i) the identification of the targeted transcript, (ii) in silico dicing of the template sequence into all possible siRNAs, (iii) calculation of the RNAi efficiency of each in silico diced siRNA, (iv) identification of sequences (siRNAs) that potentially target additional genes and (v) design of optimized PCR primers to amplify dsRNA templates directly from genomic DNA or cDNA. When predesigned dsRNAs from publicly available RNAi libraries are available, the web application retrieves the information from a relational database. Although our web application mainly aims to create optimal probes for RNAi experiments in Drosophila and C.elegans, it can also be used to create long dsRNAs that can be 'diced' in vitro to be used in mammalian cells. A general outline of the program is shown in Figure 1.

\section{User input}

Three different run-time options are available in E-RNAi. The user can choose to design RNAi probes de novo, to retrieve predesigned probes or to evaluate an input sequence for its RNAi specificity and efficiency using transcriptome databases from Drosophila, C.elegans or human. The user can also deselect database and off-target evaluation if genomic and transcript information is not available. Other options for the de novo design include siRNA length for in silico dicing (default $21 \mathrm{bp}$ ) and primer design (primer size and primer product size). The number of primer pairs to be designed is crucial for probe optimization. A higher number of designed primer pairs increases the probability of identifying an optimal probe for a specific gene but comes at a cost in terms of computing time. The 'probe retrieval' option uses nucleotide as well as amino acid sequences as input and retrieves predesigned dsRNAs from publicly available RNAi libraries of Drosophila and C.elegans. The 'probe evaluation' option runs a specificity and efficiency evaluation using the sequence information entered (Figure 2).

\section{Identification of primary targets}

First the sequence input is mapped to predicted transcripts using BLASTN against all predicted transcripts of the organism chosen by the user. This 'primary' target transcript is necessary to define which other genes are hit by the designed long dsRNA and to identify predesigned dsRNAs that are available in public libraries.

\section{In silico dicing}

Using a user-defined length, the program cuts the sequence into 18-25 nt long siRNAs with a 1 nt shifting window.

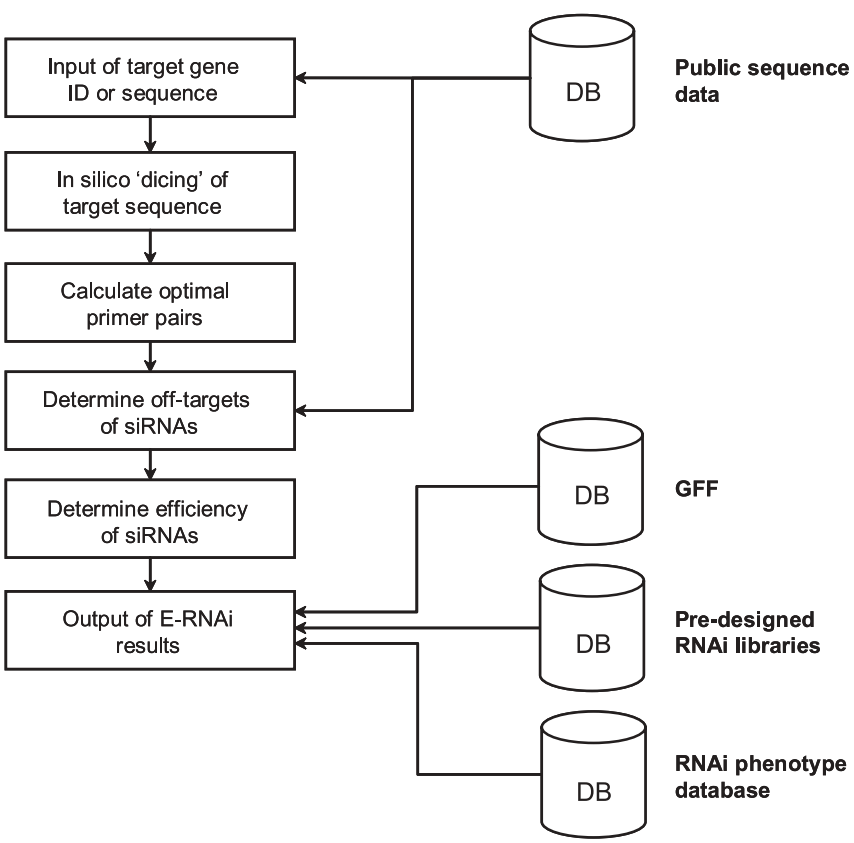

Figure 1. Schematic representation of the program. The web application was implemented as Perl scripts and CGI modules that relied on BioPerl 1.5 (26). The data are in parts, stored in a relational MySQL database. Sequence homology searches are performed using BLAST (27). Primers to amplify PCR templates for dsRNAs are automatically designed using a local implementation of Primer3 (28). Genes and RNA templates are visualized in their genomic context using the Generic Genome Browser (29). Several data sources were integrated and are accessible through E-RNAi, including transcript and gene data obtained from Flybase [Drosophila, (30)] and Wormbase [C.elegans, (31)] and predesigned RNAi libraries $(9,24)$. RNAi phenotypes were collected from Flybase, Wormbase and recent publications and assembled into a relational database that allows cross-species phenotype comparisons (unpublished data). In addition to the procedure shown here, the user can choose the option "probe retrieval' to retrieve predesigned dsRNAs. When the 'probe evaluation' option is selected, the full-length input sequence is evaluated for efficiency and specificity parameters.

The specificity and efficiency of predicted siRNAs are then calculated for these in silico diced sequences.

\section{Calculation of siRNA efficiency}

The program predicts siRNA efficiency using an algorithm described by Reynolds et al. (22). The siRNAs are evaluated for GC content, low stability at the sense strand 3 -terminus, inverted repeats and base preferences (at positions 3, 10, 13 and 19 of the sense strand). The implemented algorithm estimates the efficiency of siRNAs using eight criteria: (i) low GC content (30-52\%), (ii) at least three A/U bases at positions 15-19, (iii) absence of internal repeats, (iv) an A base at position 19, (v) an A base at position 3, (vi) a U base at position 10, (vii) a base other than $\mathrm{G}$ or $\mathrm{C}$ at position 19 and (viii) a base other than $\mathrm{G}$ at position 13. If an siRNA fulfills criteria (i), (iii), (v) and (vi), one point is added to its score. For a failure to fulfill criteria (vii) and (viii), one point is subtracted from the score. For criterion (ii), one point is added for each A or U base in positions 15-19, up to a maximum of five points. For criterion (iv), potential hairpin structures of siRNAs are calculated using RNAfold (23). If the melting temperature of the potential hairpin region is $20^{\circ} \mathrm{C}$ or less, one point is added the score. A siRNA with an efficiency score of six or higher is 


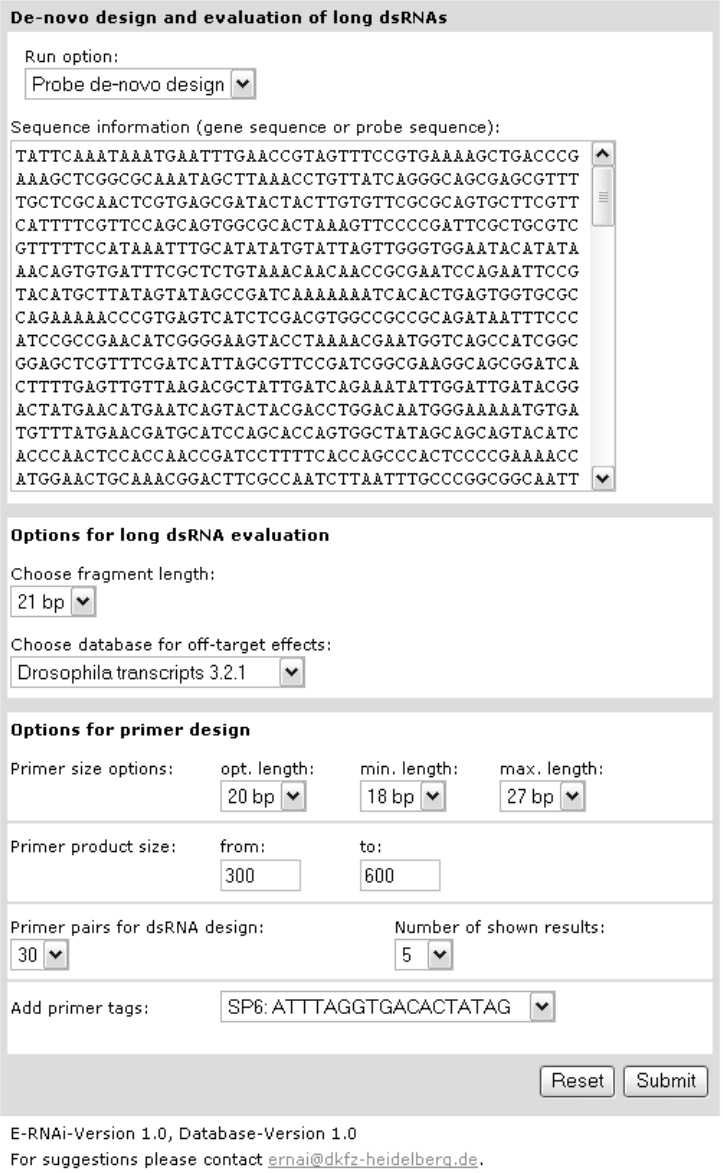

Figure 2. Input options for E-RNAi. Shown here is the input form of E-RNAi. The user is asked to choose a run option (de novo design, probe retrieval or probe evaluation) and to identify the organism for which to predict probe specificity. Users can also set the number of designed primers to be evaluated and of final results shown in the output. There is also an option to add SP6 or T7 promoter sequences to the designed primer pairs.

considered an efficient silencer (22). In the output, the percentage efficiency of a probe is calculated as the percentage of efficient siRNAs in a dsRNA.

\section{Calculation of siRNA specificity}

E-RNAi predicts the specificity of a dsRNA by performing BLAST searches of in silico diced siRNAs against the selected transcriptome using a penalty for nucleotide mismatch of -3 . BLAST searches are performed with standard settings without sequence filtering. The specificity score for an in silico diced siRNA is defined as one divided by the number of genes that are hit by a specific siRNA, whereby a hit is defined as a perfect match to a gene sequence. The complete dsRNA is then scored for an average percentage specificity for all possible siRNAs. The specificity of an RNAi construct for a certain gene and its alternative splice variants is calculated as the number of matching siRNAs over the number of all siRNAs in the dsRNA of interest.

\section{Display and export of results}

Figure 3 shows a typical result for RNAi probe de novo design. In the first table, the de novo designed RNAi probes are sorted by their overall score. This score is calculated by ranking their primer quality, their efficiency and their specificity. The weighted sum of primer quality, specificity and efficiency rankings leads to the final score and sorting of probes. Figure 3B shows an example of the RNAi probes retrieved from RNAi libraries which also target the queried gene.

\section{RNAi libraries}

The current database (version 1.0) contains RNAi probes from libraries that were designed to cover almost all open reading frames (ORFs) in the genomes of Drosophila and C.elegans. The Heidelberg/Boston RNAi library contains 21300 dsRNAs that target almost all predicted genes in the Drosophila genome $(9,24)$. About 13100 probes are available in the MRC/ Cyclacel library, which was designed on the basis of the Berkeley Drosophila Genome Project (BDGP) annotations, and cover $\sim 90 \%$ of the Drosophila genome. Predesigned probes can also be retrieved from an RNAi library of 18041 dsRNAs covering $87 \%$ of the C.elegans genome $(5,25,32)$. Additional libraries will be added as they become publicly available.

\section{EVALUATION OF LARGE-SCALE LIBRARIES}

Genome-wide RNAi libraries have been successfully used in C.elegans and Drosophila to systematically identify components of various cellular pathways. In order to benchmark individual dsRNA constructs that are part of genome-wide RNAi libraries, we applied the approach implemented in the E-RNAi software to evaluate RNAi libraries for both their predicted efficiency and their predicted specificity. Figure 4 shows the results calculated for the Heidelberg/Boston RNAi library, which targets almost every gene in the Drosophila genome $(9,24)$. The RNAi probes contained in the library are homologous to a total of 12929 genes in the Drosophila genome (25). In total, 5760284 siRNAs were computationally generated by in silico dicing using a length window of $21 \mathrm{nt}$. For each individual siRNA, we calculated efficiency and specificity scores according to the algorithms outlined above. The efficiency scores of the predicted siRNAs follow a normal distribution, with a mean efficiency score of 3.6 and a standard deviation of 2.0. In total, $18.8 \%$ of all siRNAs obtained a score $\geqslant 6$, indicating that they are predicted to be efficient silencers. We then calculated the percentage of efficient siRNAs (with a score $\geqslant 6)$ per dsRNA construct. The distribution in Figure 4C shows that 5682 out of 14300 dsRNAs contain $20 \%$ or more efficient siRNAs, whereas $3.5 \%$ have $<5 \%$ predicted efficient siRNAs. Overall, the analysis shows that an RNAi probe with an average size of $402 \mathrm{bp}$ contains 75 siRNAs that are predicted to be efficient silencers.

We then evaluated the specificity of all dsRNAs contained in the library by BLAST analysis of each individual siRNA against the Drosophila transcriptome. Of the 5641589 evaluated siRNAs, $101203(1.8 \%)$ showed more than one hit in the transcriptome. In total, 2715 dsRNAs contained at least one siRNA that potentially targets an unintended transcript. We then assessed how many off-target siRNAs can be considered to be efficient. About $84 \%$ of all cross-specific siRNAs were inefficient in silencing genes. Combining both efficiency and specificity analysis allows the conclusion that up to 


\section{A Summary}

De-novo designed RNAi probes

\begin{tabular}{|l|c|c|c|c|}
\hline Probe name & Length [bp] & Probe-specificity [\%] & Efficiency [\%] & primer3 ranking \\
\hline RNAi-probe 1 & 308 & 100.00 & 31.94 & 5 \\
\hline RNÄi-probe 2 & 381 & 100.00 & 32.69 & 23 \\
\hline RNÄi-probe 3 & 468 & 100.00 & 31.70 & 15 \\
\hline RNAi-probe 4 & 470 & 100.00 & 30.89 & 17 \\
\hline RNAi-probe 5 & 545 & 100.00 & 29.33 & 22 \\
\hline
\end{tabular}

Gbrowse image

B RNAi probes retrieved from libraries

\begin{tabular}{|c|c|c|c|c|}
\hline Probe name & Length [bp] & Probe-specificity [\%] & Efficiency [\%] & Efficient siRNAs \\
\hline$\underline{\text { HFA16819 }}$ & 497 & 100.00 & 21.80 & $104 / 477$ \\
\hline MRC012 H03 & 509 & 100.00 & 20.25 & $99 / 489$ \\
\hline
\end{tabular}

C RNÄi-probe 1

Primer information

\begin{tabular}{|c|c|c|c|c|c|}
\hline primer tags + Primer (forward/reverse, $\mathbf{5}^{\prime} \mathbf{- 3}^{\prime}$ ) & Start & Length & Tm [C] & GC [\%] & Product length [bp] \\
\hline attaggtgacactatagCGCAAACTTATCGAGCACAA & 2102 & 20 & 60.014 & 45.000 & 308 \\
\hline attaggtgacactatagACCTGTATCGTCTGGATGGC & 2409 & 20 & 59.957 & 55.000 & 308 \\
\hline
\end{tabular}

Probe sequence (308 bp)

> RNAi-probe_1

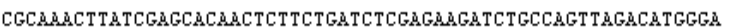

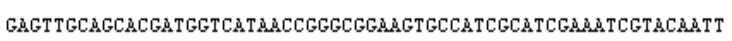

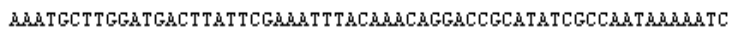

TCGCACCACAMAGTGGAGAGTGGTTTATCGAGCATGCTCTGAMTACTACAMTAGAGAC

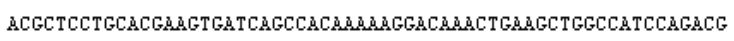

ATACAGGT

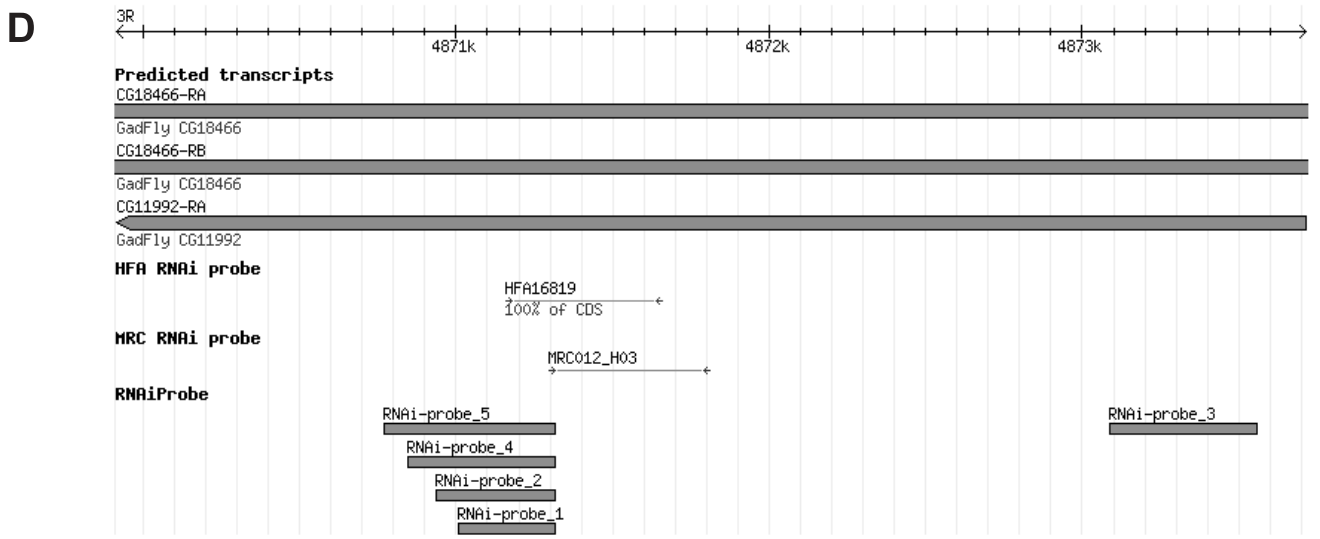

Figure 3. Output of the E-RNAi software. (A) De novo designed RNAi probes against the Rel gene are sorted by their overall score. In order to calculate this score, primer quality is weighted with 0.2 , specificity with 0.25 and efficiency with 1 . The table contains the length of each probe, the specificity and efficiency of each probe and the quality of primer pairs. (B) RNAi probes retrieved from RNAi libraries designed against the query are listed and evaluated. (C) Detailed information about each de novo designed probe is provided in the output page. A primer sequence with the SP6 promoter tag sequence (written in small letters), primer properties and probe sequence are shown. (D) De novo designed and retrieved probes are displayed on a GBrowse image at the bottom of the output page.

$1083(7.5 \%)$ of all dsRNAs contained in the Heidelberg/ Boston RNAi library could have off-target effects.

The analysis of RNAi libraries showed that a large majority of RNAi probes are predicted to be specific, and 'natural pools' of diced dsRNA give rise to many efficient siRNAs that are likely to be sufficient to knock down the target transcript. Predicted problematic probes can be computationally 'flagged' in the downstream analysis of phenotypes. 
A

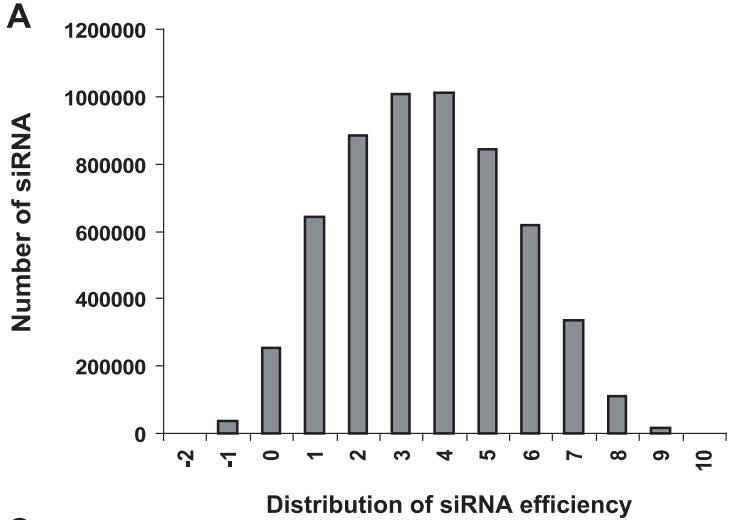

C

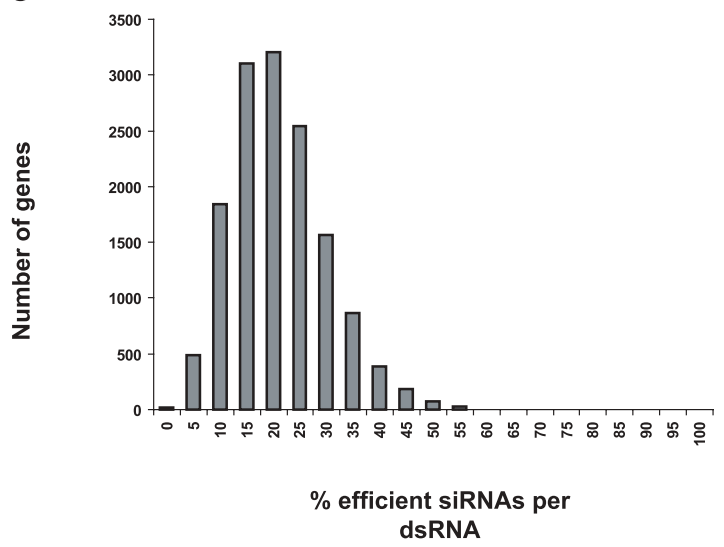

B

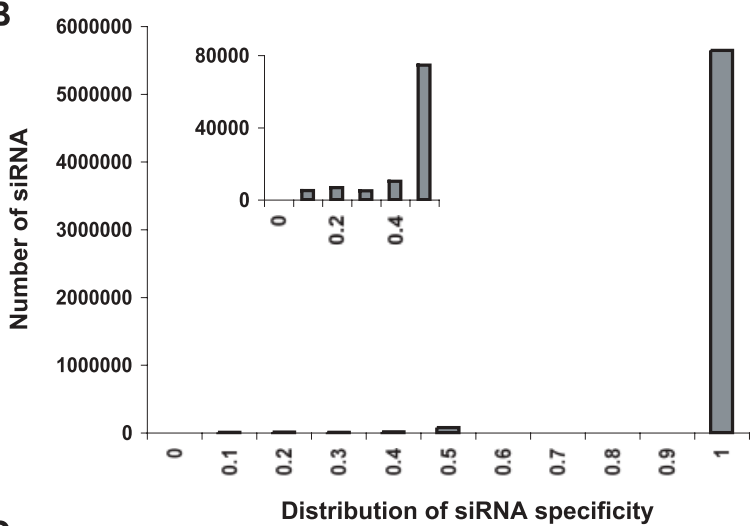

D

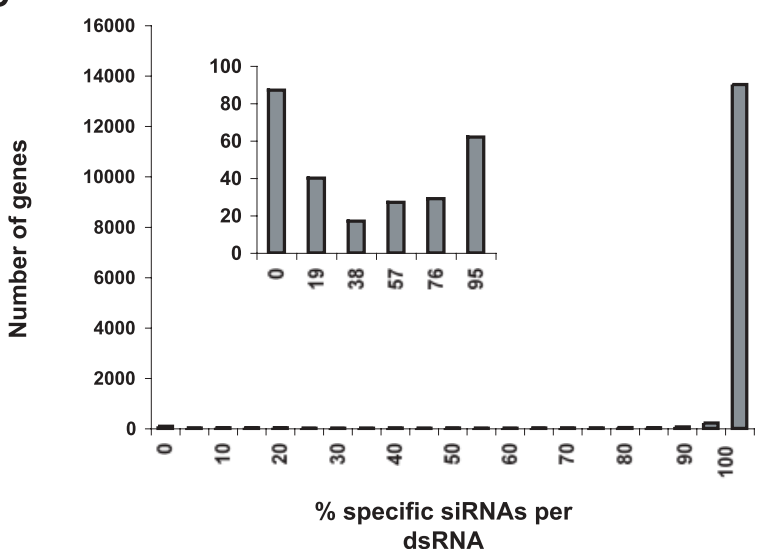

Figure 4. Analysis of genome-wide RNAi libraries. The Heidelberg/Boston RNAi library is evaluated for predicted efficiency and specificity of both individual siRNAs and dsRNAs. (A) Distribution of all siRNAs according to efficiency scores showed a mean score of 3.6. About $18.8 \%$ with an efficiency score $\geqslant 6$ are considered efficient. (B) Over 90\% of siRNAs are specific according to the specificity distribution. (C) The percentage of efficient siRNAs per gene and (D) The percentage of specific siRNAs for a single dsRNA.

A

Rel (CG11992)

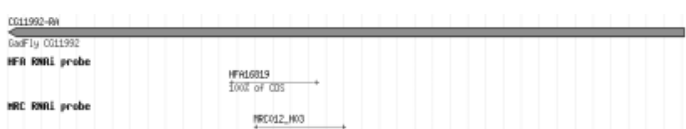

B

Efficient siRNAs

\section{Ш |||||||||||||||||||||||||||||||||||||||||||||||||||||| 7 or higher DIIII | 6 or higher}

C

Unspecific siRNAs

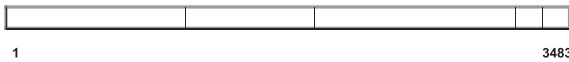

D Mask (CG 33106)

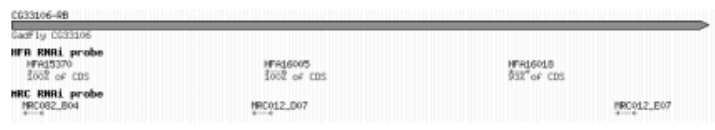

E

Efficient siRNAs

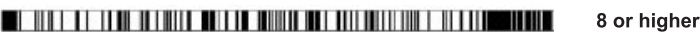
|

F Unspecific siRnAs

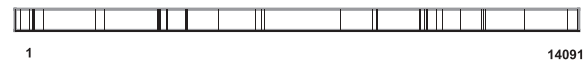

Figure 5. De novo design of optimized constructs for Rel and Mask. The coding regions of Rel (A) and Mask (D) were evaluated for specificity and efficiency in order to identify regions suitable for dsRNA design for RNAi experiments. All possibly efficient siRNAs in Rel (B) and Mask (E) are shown. Unspecific siRNAs in Rel (C) and Mask (F) are plotted to evaluate the possible off-target effects.

\section{Examples: Rel and Mask genes}

To demonstrate the functionality of the E-RNAi web application for de novo design approaches, computational predictions of dsRNA templates for two transcripts were generated. The two transcripts were chosen because of their divergent properties. Rel is a transcription factor important for innate immune responses and is encoded by a compact gene in the Drosophila genome (Figure 5A). In contrast, Mask shows significant 
homology on both the nucleotide and the protein level to other Ankyrin-domain containing genes (Figure 5D). As input, E-RNAi was given the complete ORF of Rel and Mask for the de novo design of RNAi probes. Figure 5 shows the analysis of both transcripts for efficiency and specificity scores. Approximately $22.9 \%$ of the possible siRNAs against the Rel gene show an efficiency score of 6 or higher (Figure 5B). Specific dsRNAs can be designed for most regions of the Rel gene which contains only four unspecific siRNAs, whereas a dsRNA targeting the complete ORF of the Mask gene would contain 192 unspecific siRNAs (Figure 5C and F). About $65 \%$ of unspecific siRNAs hit more than 2 genes, and $40 \%$ hit more than 10 genes. About $19.2 \%$ of the Mask transcripts are suitable for efficient gene silencing (Figure 5E). Those inefficient and unspecific regions are dispersed along the gene, which can be problematic for the design of RNAi probes.

The analysis showed that some gene regions are more prone to yield unspecific or inefficient RNAi probes than others. A dsRNA against a gene such as Mask can result in unintended gene silencing because of stretches with high sequence homology. Therefore predicted efficiency and specificity scores should play an important role during dsRNA design in order to achieve high efficiency and minimum off-target effects.

\section{DISCUSSION}

Evaluation and de novo design of long dsRNAs for efficiency and specificity remains an important issue in the design of large-scale RNAi experiments both in model organisms and in human cells. The design of long dsRNAs has to take into account both restrictions based on the properties of the contained siRNAs, such as their specificity and efficiency, and experimental limitations, such as the identification of primer pairs that are necessary to amplify the dsRNA template sequence by PCR from cDNA or genomic sources. Here we present a web application that automates the required tasks, from the prediction of efficient and specific target sites to the design of appropriate primer sequences. Results for a specified number of calculated dsRNAs are presented in their genomic context and the user can choose to export sequences as a tab-delimited file.

Limitations in the prediction of siRNA efficiency and specificity remain. The algorithms to calculate siRNA efficiency will probably be improved in the future as more experimental evidence to predict siRNA efficiency becomes available. Similarly, the calculation of potential off-target effects might be dependent on which specific regions of siRNAs are sufficient for silencing, and improved search algorithms for relevant sequence homologies will probably improve prediction of off-target effects. It is our goal to continuously update the E-RNAi web application to include improved algorithms.

In addition to de novo design, the systematic analysis of already available dsRNAs remains an important issue. Largescale RNAi libraries have been generated that target almost the complete transcriptome in Drosophila and C.elegans. A prediction of off-target effects and the efficiency of dsRNAs is important to assess both false positive and false negative rates in screening experiments. In particular, the systematic analysis of both positive and negative results from genome-wide RNAi experiments should benefit from the exclusion of transcripts that are targeted by off-target siRNAs or inefficient RNAi constructs.

\section{ACKNOWLEDGEMENTS}

We are grateful to Renato Paro and Marc Hild for providing sequence information for newly predicted genes. The MRC/ Cyclacel Drosophila RNAi library was obtained through MRC geneservices (Cambridge, UK). We are also grateful to David Emmert and William Gelbart for help with Flybase linkouts. We would like to thank Kerstin Bartscherer and Florian Fuchs for comments on the manuscript and members of the Boutros Laboratory for helpful suggestions and discussions. We are grateful to Tobias Reber for support in computational infrastructure. The research was funded in part by a Grant in the Emmy-Noether Program of the German Research Foundation to M.B. Funding to pay the Open Access publication charges for this article was provided by intramural funds.

Conflict of interest statement. None declared.

\section{REFERENCES}

1. Montgomery,M.K. and Fire,A. (1998) Double-stranded RNA as a mediator in sequence-specific genetic silencing and co-suppression. Trends Genet., 14, 255-258.

2. Hannon,G.J. (2002) RNA interference. Nature, 418, 244-251.

3. Fraser,A.G., Kamath,R.S., Zipperlen,P., Martinez-Campos,M., Sohrmann,M. and Ahringer,J. (2000) Functional genomic analysis of C.elegans chromosome I by systematic RNA interference. Nature, 408, 325-330.

4. Gönczy,P., Echeverri,C., Oegema,K., Coulson,A., Jones,S.J., Copley,R.R., Duperon,J., Oegema,J., Brehm,M., Cassin,E. et al. (2000) Functional genomic analysis of cell division in C.elegans using RNAi of genes on chromosome III. Nature, 408, 331-336.

5. Kamath,R.S., Fraser,A.G., Dong,Y., Poulin,G., Durbin,R., Gotta,M., Kanapin,A., Le Bot,N., Moreno,S., Sohrmann,M. et al. (2003) Systematic functional analysis of the Caenorhabditis elegans genome using RNAi. Nature, 421, 231-237.

6. Clemens,J.C., Worby,C.A., Simonson-Leff,N., Muda,M., Maehama,T., Hemmings,B.A. and Dixon,J.E. (2000) Use of double-stranded RNA interference in Drosophila cell lines to dissect signal transduction pathways. Proc. Natl Acad. Sci. USA, 97, 6499-6503.

7. Ramet,M., Manfruelli,P., Pearson,A., Mathey-Prevot,B. and Ezekowitz,R.A. (2002) Functional genomic analysis of phagocytosis and identification of a Drosophila receptor for E.coli. Nature, $\mathbf{4 1 6}$ 644-648.

8. Lum,L., Yao,S., Mozer,B., Rovescalli,A., Von Kessler,D., Nirenberg,M. and Beachy,P.A. (2003) Identification of Hedgehog pathway components by RNAi in Drosophila cultured cells. Science, 299, 2039-2045.

9. Boutros,M., Kiger,A.A., Armknecht,S., Kerr,K., Hild,M., Koch,B., Haas,S.A., Consortium,H.F., Paro,R. and Perrimon,N. (2004) Genome-wide RNAi analysis of growth and viability in Drosophila cells. Science, 303, 832-835.

10. Eggert,U.S., Kiger,A.A., Richter,C., Perlman,Z.E., Perrimon,N., Mitchison,T.J. and Field,C.M. (2004) Parallel chemical genetic and genome-wide RNAi screens identify cytokinesis inhibitors and targets. PLoS Biol., 2, e379.

11. Echard,A., Hickson,G.R., Foley,E. and O'Farrell,P.H. (2004) Terminal cytokinesis events uncovered after an RNAi screen. Curr. Biol., 14, 1685-1693.

12. Foley,E. and O'Farrell,P.H. (2004) Functional dissection of an innate immune response by a genome-wide RNAi screen. PLoS Biol., 2, E203.

13. Bettencourt-Dias,M., Giet,R., Sinka,R., Mazumdar,A., Lock,W.G., Balloux,F., Zafiropoulos,P.J., Yamaguchi,S., Winter,S., Carthew,R.W. et al. (2004) Genome-wide survey of protein kinases required for cell cycle progression. Nature, 432, 980-987. 
14. Ui-Tei,K., Naito,Y., Takahashi,F., Haraguchi,T., Ohki-Hamazaki,H., Juni,A., Ueda,R. and Saigo,K. (2004) Guidelines for the selection of highly effective siRNA sequences for mammalian and chick RNA interference. Nucleic Acids Res., 32, 936-948.

15. Dorsett,Y. and Tuschl,T. (2004) siRNAs: applications in functional genomics and potential as therapeutics. Nature Rev. Drug Discov., 3 , 318-329.

16. Schwarz,D.S., Hutvagner,G., Du,T., Xu,Z., Aronin,N. and Zamore,P.D. (2003) Asymmetry in the assembly of the RNAi enzyme complex. Cell, 115, 199-208.

17. Khvorova,A., Reynolds,A. and Jayasena,S.D. (2003) Functional siRNAs and miRNAs exhibit strand bias. Cell, 115, 209-216.

18. Naito,Y., Yamada,T., Ui-Tei,K., Morishita,S. and Saigo,K. (2004) siDirect: highly effective, target-specific siRNA design software for mammalian RNA interference. Nucleic Acids Res., 32, W124-W129.

19. Henschel,A., Buchholz,F. and Habermann,B. (2004) DEQOR: a web-based tool for the design and quality control of siRNAs. Nucleic Acids Res., 32, W113-W120.

20. Cui,W., Ning,J., Naik,U.P. and Duncan,M.K. (2004) OptiRNAi, an RNAi design tool. Comput. Methods Programs Biomed., 75, 67-73.

21. Kittler,R., Putz,G., Pelletier,L., Poser,I., Heninger,A.K., Drechsel,D., Fischer,S., Konstantinova,I., Habermann,B., Grabner,H. et al. (2004) An endoribonuclease-prepared siRNA screen in human cells identifies genes essential for cell division. Nature, 432, 1036-1040.

22. Reynolds,A., Leake,D., Boese,Q., Scaringe,S., Marshall,W.S. and Khvorova,A. (2004) Rational siRNA design for RNA interference. Nat. Biotechnol., 22, 326-330.

23. Hofacker,I.L. (2003) Vienna RNA secondary structure server. Nucleic Acids Res., 31, 3429-3431.

24. Hild,M., Beckmann,B., Haas,S., Koch,B., Solovyev,V., Busold,C., Fellenberg,K., Boutros,M., Vingron,M., Sauer,F. et al. (2003)
An integrated gene annotation and transcriptional profiling approach towards the full gene content of the Drosophila genome. Genome Biol., 5, R3.

25. Misra,S., Crosby,M.A., Mungall,C.J., Matthews,B.B., Campbell,K.S., Hradecky,P., Huang,Y., Kaminker,J.S., Millburn,G.H., Prochnik,S.E. et al. (2002) Annotation of the Drosophila melanogaster euchromatic genome: a systematic review. Genome Biol., 3, RESEARCH0083.

26. Stajich,J.E., Block,D., Boulez,K., Brenner,S.E., Chervitz,S.A., Dagdigian,C., Fuellen,G., Gilbert,J.G., Korf,I., Lapp,H. et al. (2002) The Bioperl toolkit: Perl modules for the life sciences. Genome Res., 12, 1611-1618.

27. Altschul,S.F., Gish,W., Miller,W., Myers,E.W. and Lipman,D.J. (1990) Basic local alignment search tool. J. Mol. Biol., 215, 403-410.

28. Rozen,S. and Skaletsky,H. (2000) Primer3 on the WWW for general users and for biologist programmers. Methods Mol. Biol., 132, $365-386$.

29. Stein,L.D., Mungall,C., Shu,S., Caudy,M., Mangone,M., Day,A., Nickerson,E., Stajich,J.E., Harris,T.W., Arva,A. et al. (2002) The generic genome browser: a building block for a model organism system database. Genome Res., 12, 1599-1610.

30. FlyBase Consortium (2003), The FlyBase database of the Drosophila genome projects and community literature. Nucleic Acids Res., 31, $172-175$.

31. Chen,N., Harris,T.W., Antoshechkin,I., Bastiani,C., Bieri,T., Blasiar,D., Bradnam,K., Canaran,P., Chan,J., Chen,C.K. et al. (2005) WormBase: a comprehensive data resource for Caenorhabditis biology and genomics. Nucleic Acids Res., 33, D383-D389.

32. Gunsalus,K.C., Yueh,W.C., MacMenamin,P. and Piano,F. (2004) RNAiDB and PhenoBlast: web tools for genome-wide phenotypic mapping projects. Nucleic Acids Res., 32, D406-D410. 